\title{
Pancreatitis, Panniculitis, and Polyarthritis Syndrome Presenting With Gouty Arthritis: A Case Report
}

\author{
Chun-Feng Lin ${ }^{\mathrm{a}}$, Ying-Ming Chiu ${ }^{\mathrm{a}, \mathrm{b}}$
}

\begin{abstract}
Pancreatitis, panniculitis, and polyarthritis (PPP) syndrome combined with gouty arthritis has never been reported in the literature. A 49-year-old male with a history of heavy alcohol abuse for at least 20 years, chronic pancreatitis, and gouty arthritis, presented with pain, swelling, and erythema involving the right thumb, right 4th metatarsophalangeal (MTP) joint, and left heel for one week. Following examination of the synovial fluid positive for monosodium urate (MSU) crystals, he was treated for gouty arthritis and the polyarthritis progressed. A skin biopsy revealed pancreatic panniculitis. Abdominal computed tomography (CT) also showed swelling of the pancreas with peripancreatic fat infiltrations. The secondary arthrocentesis was performed and the synovial fluid showed amylase 1,970 U/L, lipase 1,260 U/L, cholesterol $79 \mathrm{mg} / \mathrm{dL}$, and triglycerides $78 \mathrm{mg} / \mathrm{dL}$. We report this case to remind physicians that the first manifestation of PPP syndrome can be mistaken for gouty arthritis.
\end{abstract}

Keywords: Pancreatitis; Panniculitis; Polyarthritis; Gouty arthritis

\section{Introduction}

Pancreatitis-induced panniculitis is an uncommon condition that affects $2 \%$ to $3 \%$ of patients with pancreatic disease. When polyarthritis is also present, this represents a rare disease known as pancreatitis panniculitis polyarthritis (PPP) syndrome. Due to the absence of abdominal symptoms in

Manuscript accepted for publication March 16, 2011

${ }^{a}$ Division of Allergy, Immunology and Rheumatology, Changhua Christian Hospital, Changhua, Taiwan

${ }^{\mathrm{b}}$ Corresponding author: Ying-Ming Chiu, Division of Allergy, Immunology and Rheumatology, Changhua Christian Hospital, \#135

Nan-Siau Street, Changhua, 500 Taiwan. Email: 129273@cch.org.tw

doi: $10.4021 / \mathrm{jmc} 178 \mathrm{w}$ most cases of PPP syndrome, a delay in diagnosis is not unusual [1]. Alcoholism is one of the most common causes of chronic pancreatitis, and alcoholics often present with gouty arthritis. PPP syndrome in these patients may therefore be misdiagnosed. We report a case of arthritis being initially treated as gouty arthritis before the correct diagnosis of PPP syndrome was established.

\section{Case Report}

A 49-year-old male with a history of heavy alcohol abuse for at least 20 years, chronic pancreatitis with many acute episodes over 5 years, and gouty arthritis, presented with pain, swelling, and erythema involving the right thumb, right 4th metatarsophalangeal (MTP) joint, and left heel (Fig. 1) for one week.

Colchicine $0.5 \mathrm{mg}$ PO twice a day and methylprednisolone $40 \mathrm{mg}$ IV every 8 hours were administered initially for presumed gouty arthritis; unfortunately, the symptoms progressed. The right knee, right heel, and left 3rd and 5th MTP joints became painful, swollen, and erythematous. The right knee joint was aspirated, and examination of the synovial fluid was positive for monosodium urate (MSU) crystals.

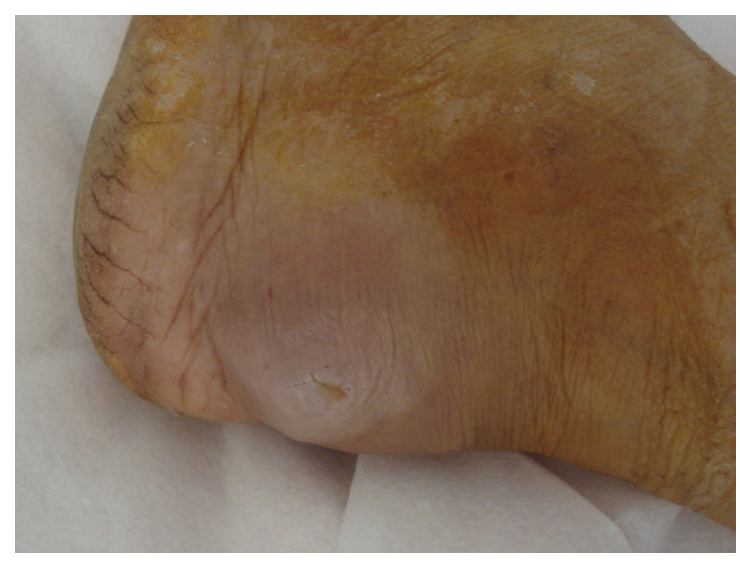

Figure 1. Swelling and erythema of the left heel with pus formation. 


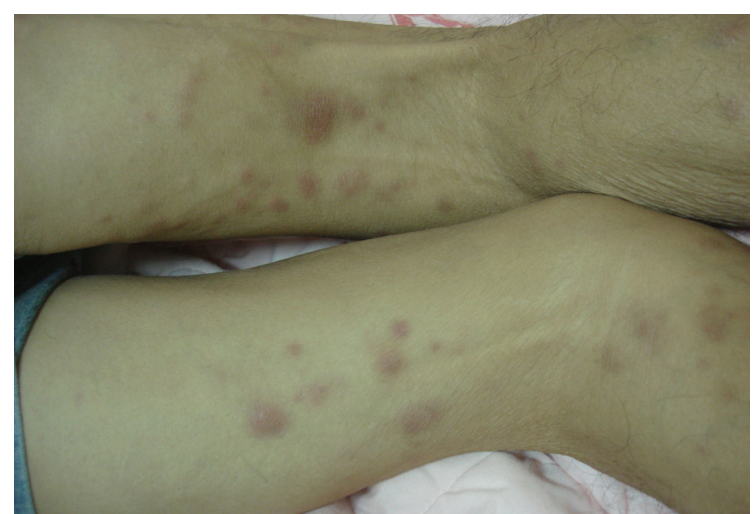

Figure 2. Erythematous nodules over the posterior thighs bilaterally.

Colchicine $0.5 \mathrm{mg}$ PO twice a day and methylprednisolone $40 \mathrm{mg}$ IV every 8 hours were continued; however, purulent fluid developed over the right thumb, right 4th MTP joint, left heel, and dorsum of the feet bilaterally. Aspiration was performed from left heel, followed by administration of oxacillin 2,000 mg IV every 6 hours. The fluid culture grew Escherichia coli, so the antibiotics were changed to gentamicin $80 \mathrm{mg}$ IV once a day and cefazolin $1 \mathrm{~g}$ IV every 8 hours; methylprednisolone was tapered to $40 \mathrm{mg}$ IV once a day. However, the patient developed a fever, and purulent fluid continued to accumulate in bilateral knees, right thumb, right 4th MTP joint, left heel and dorsum of the feet bilaterally. Arthrocentesis was again performed from right knee, and synovial fluid examination this time failed to show MSU or calcium pyrophosphate crystals. The synovial fluid culture was negative. Due to an elevated white blood cell count (WBC 22,500/ $\mu \mathrm{L}$ ) with a left shift (neutrophils 90.1\%) and a C-reactive protein (CRP) of $15.4 \mathrm{mg} / \mathrm{dL}$, cefazolin was changed to ampicillin-sulbactam $1.5 \mathrm{~g}$ IV every 6 hours. Methylprednisolone was tapered and switched to oral prednisolone $5 \mathrm{mg}$ twice a day. Gentamicin $80 \mathrm{mg}$ IV once a day and colchicine $0.5 \mathrm{mg}$ PO twice a day were continued. Fever persisted, and new erythematous nodules formed on the thighs and upper arms bilaterally (Fig. 2). A skin biopsy was then performed. While awaiting the pathology report, the antibiotics were changed to cefpirome, and prednisolone and colchicine were continued. The right thumb required debridement due to the persistence of purulent fluid, and vancomycin was prescribed. The skin biopsy pathology report showed fat necrosis as well as ghost cells, which suggested pancreatic panniculitis.

After the pathology report, serum amylase and lipase levels were 4,840 U/L and 7,200 U/L, respectively. An abdominal computed tomography (CT) scan identified edema involving the pancreas with peripancreatic fat infiltrations. Interestingly, the patient denied abdominal pain throughout the entire hospitalization. The amylase and lipase continued to increase $(7,520 \mathrm{U} / \mathrm{L}$ and $12,200 \mathrm{U} / \mathrm{L}$, respectively). A third

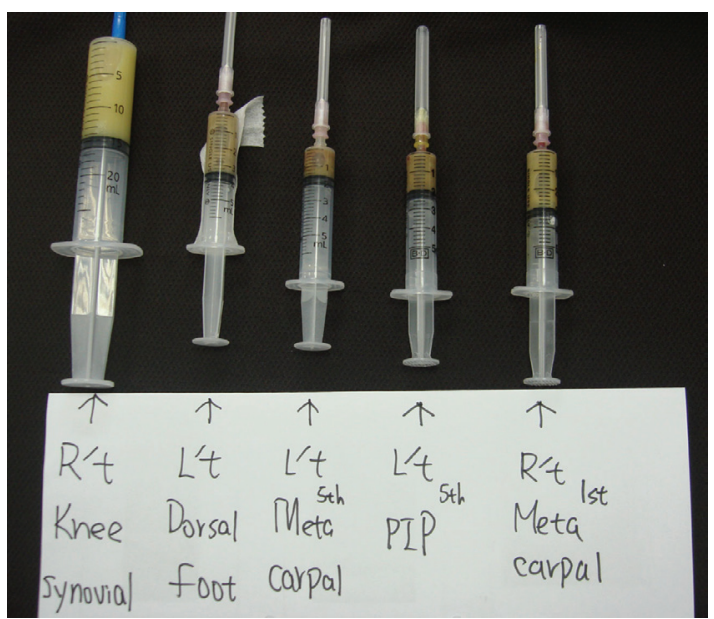

Figure 3. Right knee synovial fluid.

arthrocentesis was performed on the right knee. Synovial fluid showed amylase 1,970 U/L, lipase 1,260 U/L, cholesterol $79 \mathrm{mg} / \mathrm{dL}$, and triglycerides $78 \mathrm{mg} / \mathrm{dL}$. A Sudan stain performed on aspirate from the left heel lesion was negative. All antibiotics were discontinued, and the patient received nothing by mouth for 48 hours. He was then started on a lowfat diet; however, the serum amylase and lipase remained significantly elevated $(13,180 \mathrm{U} / \mathrm{L}$ and $21,900 \mathrm{U} / \mathrm{L}$, respectively). The erythematous nodules over the thighs and upper arms subsided gradually, and the purulent fluid accumulated over the dorsum of the feet also decreased. The patient was discharged on December 21, 2009.

\section{Discussion}

Many cases of PPP syndrome have been reported. Although there are some patients with panniculitis and elevated serum lipase levels without pancreatic disease [1], most patients with PPP syndrome have acute or chronic pancreatitis [2], and some have pancreatic tumors or ischemic disease [3, 4]. The patient discussed in the case report had a history of chronic pancreatitis with many acute flare-ups over a 5-year period.

The typical patient with PPP syndrome is a middle-aged male with heavy alcohol use. Approximately two-thirds of patients present either without abdominal symptoms or with only mild symptoms, which can lead to a delay in diagnosis or misdiagnosis and a poorer prognosis [3]. Our 49-yearold male patient with a 20-year history of heavy alcohol use matches the aforementioned criteria for PPP syndrome. The lack of abdominal symptoms combined with his history of gouty arthritis and presence of MSU crystals in his right knee synovial fluid led to an initial diagnosis of gouty arthritis. Without the presence of erythematous nodules in the first few days after admission, pancreatic polyarthritis was not 
included in the differential diagnosis.

A symmetric or an asymmetric polyarthritis that includes both the small and large joints is typical for PPP syndrome; however, a few cases of oligoarthritis or monoarthritis have also been reported $[3,5]$. Although there is no case report of coexisting gouty arthritis, septic arthritis, or rheumatoid arthritis with PPP syndrome, these diseases should all be considered in the differential diagnosis because their initial presentations may be similar.

Elevated levels of pancreatic enzymes can induce fat necrosis in the joint and adjacent periarticular tissues and lead to arthritis [6]. Thick creamy synovial fluid with a high viscosity is typical for PPP syndrome (Fig. 3). Microscopic examination can reveal birefringent crystal-like structures, which are due to the presence of lipid crystals [7]. We identified elevated pancreatic enzyme levels in synovial fluid aspirated from the knee joint and checked the cholesterol and triglyceride levels as well. However, the Sudan stain of the aspirate was negative, and microscopic examination failed to reveal evidence of lipid crystals.

The serum pancreatic enzyme level does not always correlate with the severity of the arthritis, but it does correlate with the progression of the fat necrosis [3]. However, in our case, the serum amylase and lipase levels increased gradually after PPP syndrome was diagnosed, and the arthritis and erythematous nodules slowly improved. Based on this case, amylase and lipase may not be suitable markers for the progression or improvement of PPP syndrome.

There is currently no case report with PPP syndrome coexistent with gouty arthritis, in both multiple joint involved, and we reported the case to illustrate the difficulty associated with establishing the correct diagnosis prior to the appear- ance of erythematous nodules. Physicians should consider PPP syndrome in a patient with polyarthritis and a history of pancreatitis.

\section{References}

1. Forstrom TL, winkelmann RK. Acute, generalized panniculitis with amylase and lipase in skin. Arch Dermatol 1975;111(4):497-502.

2. Francombe J, Kingsnorth AN, Tunn E. Panniculitis, arthritis and pancreatitis. Br J Rheumatol 1995;34(7):680683.

3. Narvaez J, Bianchi MM, Santo P, de la Fuente D, RiosRodriguez V, Bolao F, Narvaez JA, et al. Pancreatitis, panniculitis, and polyarthritis. Semin Arthritis Rheum 2010;39(5):417-423.

4. Smukler NM, Schumacher HR, Pascual E, Brown S, Ryan WE, Sadeghian MR. Synovial fat necrosis associated with ischemic pancreatic disease. Arthritis Rheum 1979;22(5):547-553.

5. Baron M, Paltiel H, Lander P. Aseptic necrosis of the talus and calcaneal insufficiency fractures in a patient with pancreatitis, subcutaneous fat necrosis, and arthritis. Arthritis Rheum 1984;27(11):1309-1313.

6. Mustafa KN, Hadidy A, Shoumaf M, Razzuki SA. Polyarthritis with chondronecrosis associated with osteonecrosis, panniculitis and pancreatitis. Rheumatol Int 2010;30(9):1239-1242.

7. Saag KG, Niemann TH, Warner CA, Naides SJ. Subcutaneous pancreatic fat necrosis associated with acute arthritis. J Rheumatol 1992;19(4):630-632. 